\title{
Perfil de usuários com prescrição de omeprazol em uma Unidade Básica de Saúde do Sul do Brasil: considerações sobre seu uso racional
}

\author{
Use of omeprazole at a Basic Health Unit in southern Brazil: patient profile, indication, and \\ duration of use
}
Perfil de usuario de recetas de omeprazol en una Unidad Básica de Salud en el sur de Brasil: impacto del tiempo de tratamiento y el uso racional de los medicamentos

Priscila Hipólito. Faculdade de Farmácia - Universidade Federal do Rio Grande do Sul (UFRGS). Porto Alegre, RS, Brasil. priscilahipolito@yahoo.com.br

Bruno Simas da Rocha. Seção de Farmácia Clínica - Hospital de Clínicas de Porto Alegre (HCPA). Porto Alegre, RS, Brasil. bsrocha@ hcpa.edu.br (Autor correspondente)

Francisco Jorge Arsego Quadros de Oliveira. Departamento de Medicina Social da Faculdade de Medicina - Universidade Federal do Rio Grande do Sul (UFRGS). Porto Alegre, RS, Brasil. farsego @ hcpa.edu.br

\section{Resumo}

Introdução: Os inibidores da bomba de prótons (IBPs) são uma das classes terapêuticas mais prescritas no mundo, sendo omeprazol o representante nas listas de medicamentos essenciais. Estudos indicam que o uso a curto prazo dos IBPs pode ser seguro, apesar de estar relacionado efeitos adversos a longo prazo. Objetivo: Avaliar o perfil dos usuários com prescrição de omeprazol em uma Unidade Básica de Saúde (UBS), relacionando com tempo de uso, dose e indicação. Métodos: Estudo transversal no qual foram avaliados os prontuários de usuários com prescrições de omeprazol atendidas no mês de maio de 2014 pela farmácia de uma UBS. Resultados: Foram incluídos no estudo 349 pacientes, sendo $75,4 \%$ do sexo feminino. A média de medicamentos prescritos por pacientes foi de 4,5 e a idade média de 64,6 anos. A dose de $20 \mathrm{mg}$ foi encontrada em $69,8 \%$ dos usuários, $84,3 \%$ tem prescrição mais de seis meses, e a doença do refluxo e a úlcera gástrica foram as indicações mais citadas; $29,5 \%$ dos pacientes não tinham registro de motivo de uso do omeprazol. Conclusão: O maior tempo de uso do omeprazol está associado com maior idade e número de medicamentos prescritos, podendo estes fatores estarem relacionados com o uso irracional de omeprazol, por vezes sem justificativa em prontuário e com tempo prolongado de uso.

\begin{abstract}
Introduction: Proton pump inhibitors (PPIs) are one of the most prescribed therapeutic classes worldwide. Omeprazole, a PPI, is included in the essential medicines list. Studies indicate that short-term use of PPIs can be safe, but that their long-term use is associated with adverse effects. Objective: To evaluate the profile of patients prescribed omeprazole at a Basic Health Unit (BHU) and to evaluate the duration of use, prescribed dose, and indication for use. Methods: This was a cross-sectional study. The medical records of patients prescribed omeprazole in May 2014 were identified by the pharmacy of the BHU, and were reviewed. Results: Of the 349 patients included, $75.4 \%$ were female patients and the average age was 64.6 years. The average number of drugs prescribed was 4.5 per patient. A dose of $20 \mathrm{mg}$ was prescribed most frequently $(69.8 \%)$, and $84.3 \%$ of patients had been prescribed omeprazole for more than six months. Gastro-esophageal reflux disease and gastric ulcers were the most cited indications for use. However, in $29.5 \%$ of the patients in the medical records examined, no indication for the use of omeprazole could be found. Conclusion: Older age and the number of prescription drugs were associated with increased omeprazole use. These factors can also be associated with irrational, and sometimes unjustified, use of omeprazole, and with its prolonged use.
\end{abstract}

Como citar: Hipólito $\mathrm{P}$, Rocha BS, Oliveira FJAQ. Perfil de usuários com prescrição de omeprazol em uma Unidade Básica de Saúde do Sul do Brasil: considerações sobre seu uso racional. Rev Bras Med Fam Comunidade. 2016;11(38):1-10. http://dx.doi.org/10.5712/rbmfc11(38)1153
Palavras-chave:

Omeprazol

Prescrições de Medicamentos Unidade Básica de Saúde

\section{Keywords:}

Omeprazole

Drug Prescriptions

Health Centers

Fonte de financiamento: declaram não haver.

Parecer CEP: 802.165 (HCPA/UFRGS), aprovado em 17/09/2014. Conflito de interesses: declaram não haver. Procedência e revisão por pares: revisado por pares.

Recebido em: 01/06/2015.

Aprovado em: 05/02/2016. 


\section{Resumen}

Introdución: Los inhibidores de la bomba de protones (IBP) son una de las clases terapéuticas más recetados en el mundo, y omeprazol es el representante frente a las listas de medicamentos esenciales. Los estudios indican que el uso em poco tiempo de los IBP puede ser seguro, aunque está relacionado con efectos adversos a largo plazo. Objectivo: Evaluar el perfil de los usuarios con las recetas de omeprazol en una Unidad Básica de Salud (UBS), evaluando la duración del uso, dosis e indicación. Métodos: Diseño transversal donde se evaluaron las historias clínicas de los usuarios con las recetas de omeprazol atendidas en mayo 2014 en la farmacia de UBS. Resultados: El estudio incluyó a 349 pacientes, el $75,4 \%$ eran mujeres. El número medio de fármacos prescritos para los pacientes fue de 4,5 y la edad promedio de 64,6 años. La dosis de $20 \mathrm{mg}$ se encontró en el $69,8 \%$ de los usuarios, el $84,3 \%$ tiene receta para más de seis meses, y la enfermedad de reflujo y úlceras gástricas fueron las indicaciones más citadas; $29,5 \%$ de los pacientes no tenían registro de la razón para el uso de omeprazol. Conclusión: El tiempo de mayor uso de omeprazol se asocia con la edad avanzada y el número de medicamentos recetados, y estos factores están relacionados con el uso irracional de omeprazol, a veces sin justificación en los registros médicos y el uso a largo plazo.
Palabras clave:

Omeprazol

Prescripciones de Medicamentos Centros de Salud

\section{Introdução}

Os inibidores da bomba de prótons (IBPs) são uma das classes terapêuticas mais prescritas no mundo, incluindo o uso a longo prazo, por possuir aspectos que integram alta eficácia com baixa toxicidade. ${ }^{1}$ O modo de ação desta classe ocorre pela supressão da secreção de ácido gástrico por meio de inibição

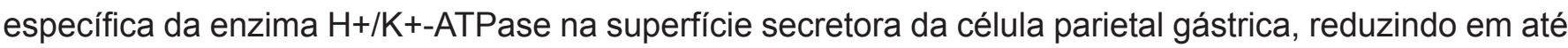
$95 \%$ a produção diária de ácido gástrico. ${ }^{2,3}$ Entre os representantes desta classe, omeprazol é o mais prescrito, ${ }^{4}$ fazendo parte da Relação Nacional de Medicamentos Essenciais do Brasil ${ }^{5}$ e da Organização Mundial da Saúde (OMS). ${ }^{6}$

Os IBPs são indicados para o tratamento da úlcera péptica associada à Helicobacter pylori, sintomas dispépticos relacionados com o uso contínuo de anti-inflamatórios não esteroides (AINEs) e ácido acetilsalicílico, dispepsia não associada à úlcera, doença do refluxo gastroesofágico (DRGE) e esofagite de refluxo, além de participarem dos esquemas de erradicação de Helicobacter pylori.2,7,8 Para o tratamento da maioria dessas doenças, geralmente é necessário um curto tempo, normalmente semanas. ${ }^{2,3}$

Alguns estudos indicam que o uso dos IBPs por longo período de tempo pode ser seguro, desde que haja acompanhamento médico. ${ }^{9}$ No entanto, outros estudos levantam questões sobre a segurança do uso contínuo de IBP no manejo de doença péptica relacionada à acidez gástrica, alertando para que a recomendação de uso seja mediante indicações precisas e períodos estabelecidos pelos prescritores. ${ }^{1,10}$

O omeprazol inibe acima de $90 \%$ da secreção ácida de 24 horas na maioria dos pacientes, tornando muitos destes pacientes quase aclorídricos, permitindo a colonização da mucosa gástrica por bactérias capazes de reduzir nitrato a nitrito, o que aumenta a produção local de nitritos. Com isso, há uma clara associação entre carcinoma gástrico e acloridria no homem e, além disso, como muitos indivíduos tratados para úlcera apresentam também alterações pré-cancerosas no estômago, é recomendável prudência no uso clínico prolongado de medicamentos que causam hipocloridria ou acloridria. ${ }^{11,12}$

Outros estudos associam o uso crônico de omeprazol com a má absorção do cálcio pelos ossos, podendo levar à osteoporose, e consequente aumento de fraturas ósseas. ${ }^{13}$ Estudos recentes também indicam que o consumo exagerado dos IBPs está diretamente ligado à deficiência do ferro e magnésio, minerais fundamentais ao metabolismo humano, que estão relacionados ao aumento da susceptibilidade à pneumonia e infecções entéricas. ${ }^{1,8,14}$ 
Em outros países, como na Espanha, estudos avaliaram a adequação da prescrição de IBPs e mostraram que $63,6 \%$ das prescrições não apresentavam indicações que justificariam o uso desta classe de medicamentos. ${ }^{15} \mathrm{Um}$ segundo estudo realizado no mesmo país avaliou a prevalência do uso de IBP em pacientes de um hospital, apontando que $77,6 \%$ das prescrições estavam não conformes. ${ }^{16}$

A prescrição de omeprazol fora das indicações estabelecidas em protocolos e diretrizes terapêuticas, como o Formulário Terapêutico Nacional, pode constituir uma não conformidade de prescrição e possível erro de medicação. ${ }^{17} \mathrm{~A}$ alegação mais frequente para uso de um IBP, no caso, omeprazol, seria para a prevenção de gastrite em pacientes com polifarmácia, ${ }^{7,17}$ que pode ser definida como o uso de múltiplos fármacos. ${ }^{18}$ Esta última indicação possui menor evidência de uso, podendo ser revista. ${ }^{19}$

Tendo em vista este panorama, o objetivo deste estudo foi avaliar o perfil dos usuários com prescrição de omeprazol na Atenção Básica, relacionando a indicação que justifique o uso deste medicamento, dose e tempo de uso do mesmo.

\section{Métodos}

Foi realizado um estudo transversal em que foram avaliados os pacientes com prescrições contendo o medicamento omeprazol, atendidos no mês de maio de 2014 pela farmácia de uma Unidade Básica de Saúde, provenientes dos prescritores desta mesma unidade. Esta UBS encontra-se numa região central do município, com cerca de 40 mil usuários adstritos e 32 mil cadastrados, com alta concentração de idosos com diversas comorbidades. Esta unidade foi escolhida para o estudo devido ao seu perfil e controle de fornecimento dos medicamentos de uso contínuo na farmácia.

Foram incluídos no estudo os usuários com prescrição de omeprazol atendidos na farmácia da UBS, desde que a receita fosse proveniente dos prescritores desta UBS, devido ao acesso dos prontuários de famílias dos mesmos. Foi escolhido aleatoriamente o mês de maio de 2014 para a coleta das receitas, tendo em vista que os medicamentos de uso contínuo são fornecidos a cada 30 dias. Foram excluídos usuários cujas receitas não foram fornecidas por médicos da UBS ou indivíduos que não possuem prontuário de família na unidade em questão.

Os dados foram coletados a partir dos prontuários destes pacientes e registrados em uma ficha de extração elaborada para este fim, contendo as seguintes variáveis: nome do paciente, número do prontuário individual, número do prontuário de família, idade, sexo, doença que justifique o uso de omeprazol, dose utilizada, tempo de uso, número de medicamentos utilizados, presença de comorbidades. Após o período de coleta, todas as informações foram digitadas em banco de dados no Microsoft Excel 2007 e a análise dos dados foi feita através do pacote estatístico Statistical Package for the Social Sciences versão 16.0. O perfil dos usuários que utilizam omeprazol foi traçado com o uso de análise descritiva, com apresentação de médias e frequências para estes dados.

A associação entre as variáveis categóricas foi realizada com o uso do teste de qui-quadrado de Pearson, e as variáveis contínuas pelo Teste t de Student, ambos com significância de a=0,05.

Este estudo foi aprovado no Comitê de Ética em Pesquisa do Hospital de Clínicas de Porto Alegre (Porto Alegre, RS). 


\section{Resultados}

Foram incluídos no estudo 349 pacientes, sendo 86 (24,6\%) do sexo masculino e $263(75,4 \%)$ do sexo feminino. A Figura 1 apresenta o fluxograma da população-alvo e seleção dos usuários para o estudo.

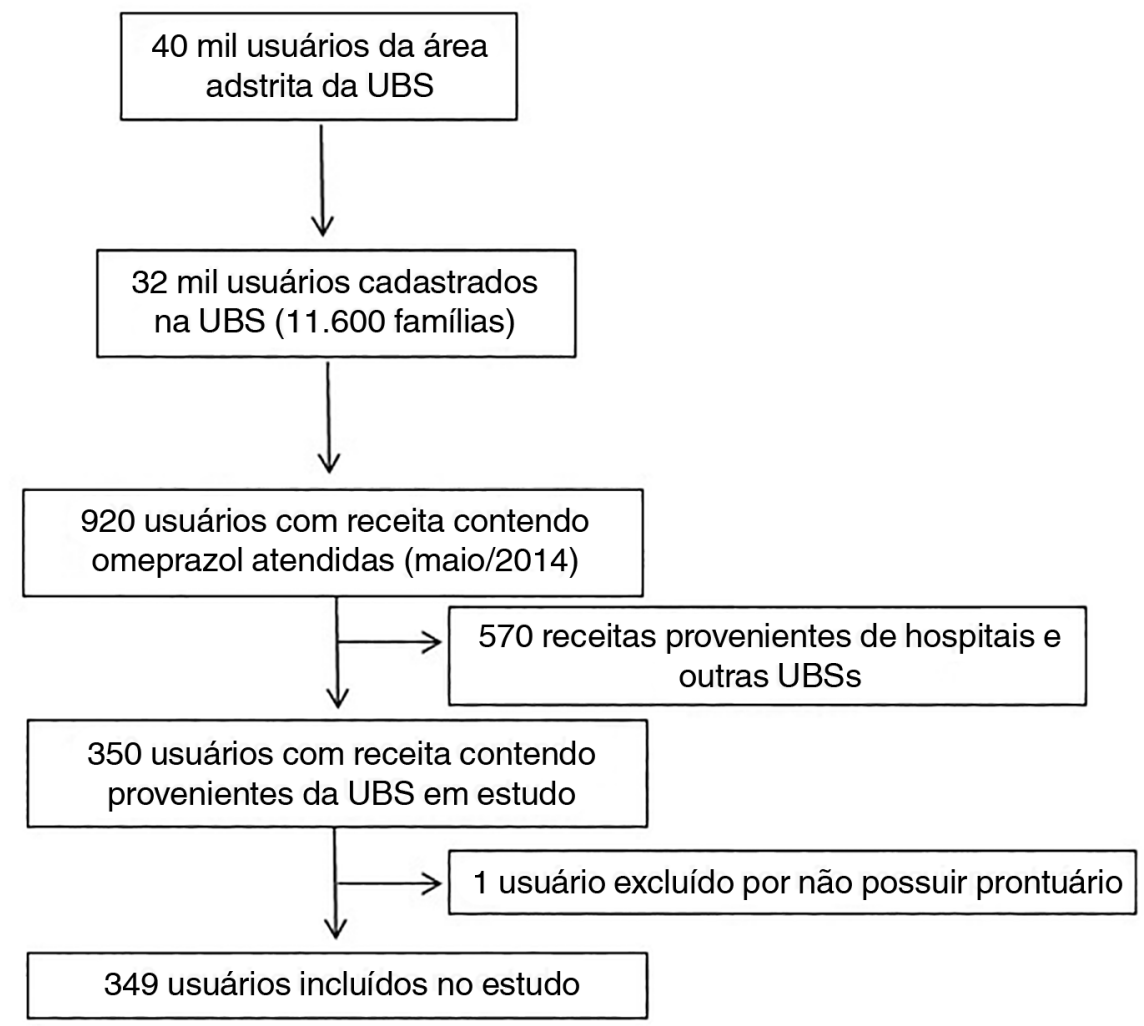

Figura 1. Fluxograma de seleção dos indivíduos para o estudo.

A Tabela 1 descreve a amostra conforme o sexo, com média de medicamentos em uso pelos pacientes de 4,5 medicamentos ( $\mathrm{DP}=1,7$ medicamentos) e a idade média foi de 64,6 anos ( $\mathrm{DP}=15,1$ anos). Os resultados desta tabela indicam que a média de medicamentos prescritos é maior no sexo feminino (4,67 medicamentos) do que no sexo masculino ( 4,02 medicamentos), sendo este dado estatisticamente significativo $(p=0,002)$.

Com relação às indicações de uso, a doença do refluxo $(28,4 \%)$ e a úlcera gástrica $(14 \%)$ foram as mais descritas nos prontuários para tratamento com o omeprazol. Outras indicações podem ser inferidas com base nos registros de sintomas relatados pelos pacientes em prontuário (azia, dispepsia, dor no estômago). Não foi encontrada informação de indicações explícitas para a prescrição de omeprazol no prontuário de $26,9 \%$ dos usuários. Aqueles que utilizam o omeprazol com prescrição médica sem nenhuma indicação descrita em prontuário representam $29,5 \%$ dos pacientes em estudo. Dos 349 usuários com omeprazol prescrito, 35,8\% possuem prescrição de ácido acetilsalicílico para prevenção de eventos cardiovasculares e 2,9\% possuem prescrição concomitante de anti-inflamatório não esteroide.

A Tabela 2 apresenta a amostra conforme a dose diária de omeprazol utilizada (20mg ou dose acima de 20mg). 
Tabela 1. Descrição da amostra conforme o sexo.

\begin{tabular}{|c|c|c|c|c|}
\hline Variável & Total n (\%) ou média (DP) & Masculino n (\%) ou média (DP) & Feminino $n(\%)$ ou média (DP) & p \\
\hline Média de idade & $64,6(15,1)$ & $63,3(16,3)$ & $65(14,7)$ & 0,369 \\
\hline Média de medicamentos & $4,5(1,7)$ & $4,02(1,9)$ & $4,67(1,6)$ & $0,002^{*}$ \\
\hline Faixa etária & & & & 0,501 \\
\hline 0 a 10 anos & $1(0,3 \%)$ & 0 & $1(0,4 \%)$ & \\
\hline 11 a 20 anos & $3(0,9 \%)$ & $1(1,2 \%)$ & $2(0,8 \%)$ & \\
\hline 21 a 30 anos & $8(2,3 \%)$ & $2(2,3 \%)$ & $6(2,3 \%)$ & \\
\hline 31 a 40 anos & $18(5,2 \%)$ & $8(9,3 \%)$ & $10(3,8 \%)$ & \\
\hline 41 a 50 anos & $24(6,9 \%)$ & $6(7,0 \%)$ & $18(6,8 \%)$ & \\
\hline 51 a 60 anos & $66(18,9 \%)$ & $13(15,1 \%)$ & $53(20,2 \%)$ & \\
\hline 61 a 70 anos & $104(29,8 \%)$ & $29(33,7 \%)$ & $75(28,5 \%)$ & \\
\hline 71 anos ou mais & $125(35,8 \%)$ & $27(31,4 \%)$ & $98(37,3 \%)$ & \\
\hline Justificativa em prontuário & $246(70,5 \%)$ & $57(66,3 \%)$ & $189(71,9 \%)$ & 0,495 \\
\hline Indicação de uso & & & & 0,645 \\
\hline Doença do refluxo & $99(28,4 \%)$ & $19(22,1 \%)$ & $80(30,4 \%)$ & \\
\hline Úlcera gástrica & $49(14,0 \%)$ & $15(17,4 \%)$ & $34(12,9 \%)$ & \\
\hline Esofagite erosiva & $2(0,6 \%)$ & 0 & $2(0,8 \%)$ & \\
\hline Úlcera duodenal & $1(0,3 \%)$ & 0 & $1(0,4 \%)$ & \\
\hline Doença por Helicobacter pylori & $1(0,3 \%)$ & 0 & $1(0,4 \%)$ & \\
\hline Outras indicações ** & $94(26,9 \%)$ & $24(27,9 \%)$ & $70(26,6 \%)$ & \\
\hline Sem justificativa & $103(29,5 \%)$ & $28(32,6 \%)$ & $75(28,5 \%)$ & \\
\hline Dose utilizada & & & & 0,788 \\
\hline $20 \mathrm{mg}$ & $243(69,8 \%)$ & $57(66,3 \%)$ & $186(71,0 \%)$ & \\
\hline $40 \mathrm{mg}$ & $91(26,1 \%)$ & $26(30,2 \%)$ & $65(24,8 \%)$ & \\
\hline $60 \mathrm{mg}$ & $10(2,9 \%)$ & $2(2,3 \%)$ & $8(3,1 \%)$ & \\
\hline $80 \mathrm{mg}$ & $4(1,1 \%)$ & $1(1,1 \%)$ & $3(1,1 \%)$ & \\
\hline Tempo de uso de omeprazol & & & & 0,726 \\
\hline 0 a 30 dias & $23(6,6 \%)$ & $7(8,1 \%)$ & $16(6,1 \%)$ & \\
\hline 30 a 90 dias & $17(4,9 \%)$ & $5(5,8 \%)$ & $12(4,6 \%)$ & \\
\hline 3 a 6 meses & $7(2,0 \%)$ & $1(1,2 \%)$ & $6(2,3 \%)$ & \\
\hline 6 meses a um ano & $46(13,2 \%)$ & $13(15,1 \%)$ & $33(12,5 \%)$ & \\
\hline 1 a 2 anos & $40(11,5 \%)$ & $6(7,0 \%)$ & $34(12,9 \%)$ & \\
\hline 2 a 5 anos & $93(26,6 \%)$ & $25(29,1 \%)$ & $68(25,9 \%)$ & \\
\hline 5 anos ou mais & $90(25,8 \%)$ & $19(22,1 \%)$ & $71(27,0 \%)$ & \\
\hline Sem descrição no prontuário & $32(90,2 \%)$ & $10(11,6 \%)$ & $22(8,4 \%)$ & \\
\hline
\end{tabular}

Conforme apresentado na Tabela 2, há diferença estatisticamente significativa entre os pacientes que têm justificativa médica para o uso de omeprazol $(p=0,007)$, em que um número maior de pacientes com dose superior a $20 \mathrm{mg}$ possui justificativa em prontuário.

A Tabela 3 apresenta a descrição da amostra conforme o tempo de uso de omeprazol. Entre os usuários com descrição de tempo de uso de omeprazol, $84,3 \%$ usam há mais de seis meses. A média de idade dos pacientes que utilizam omeprazol há mais de seis meses foi de 66,3 anos, e dos pacientes que 
Tabela 2. Descrição da amostra conforme a dose diária de omeprazol utilizada (20mg ou mais).

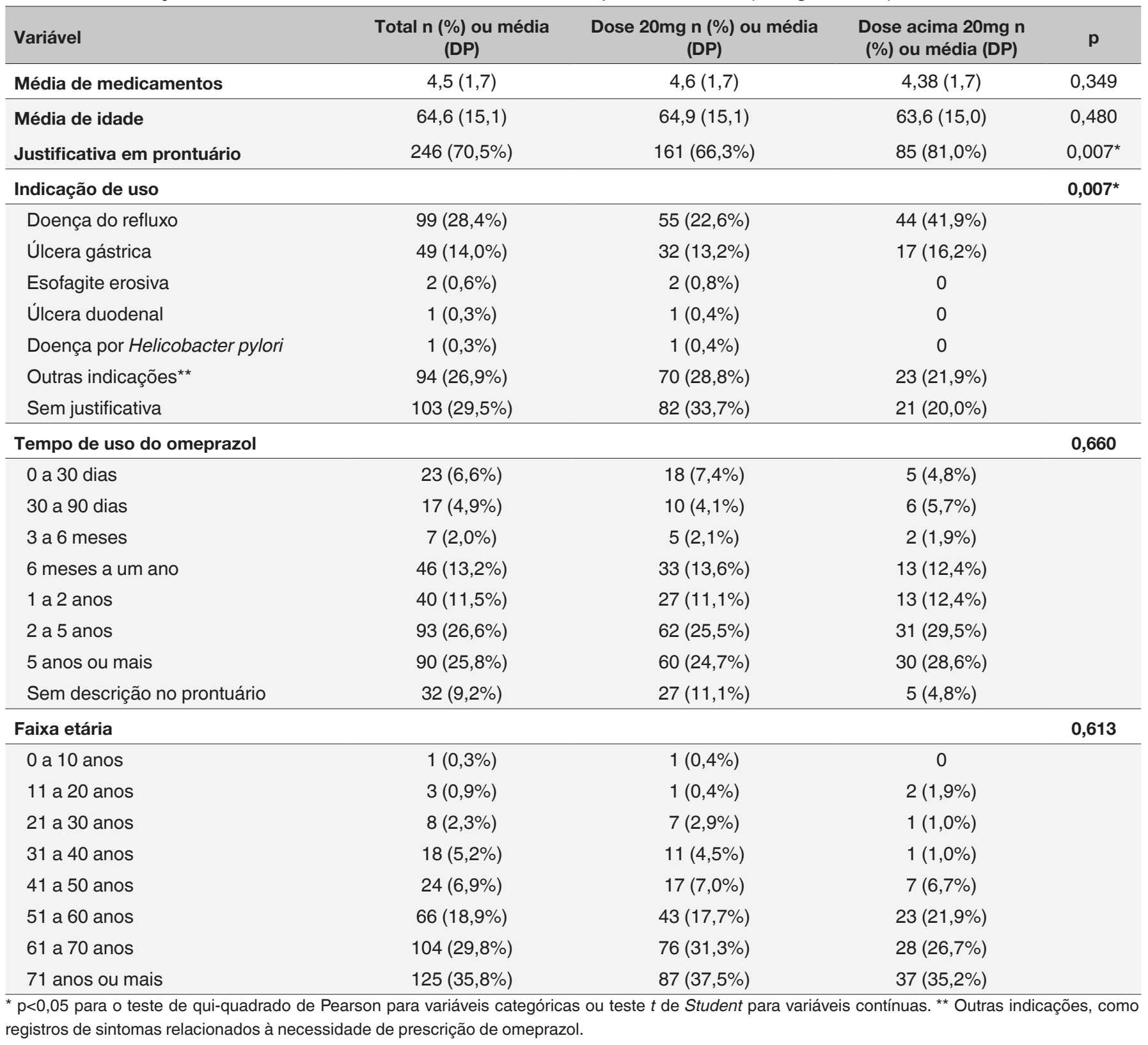

utilizam este medicamento há seis meses ou menos foi de 59,3 anos, sendo esta diferença estatisticamente significativas $(p=0,003)$.

Observando-se os dados da tabela, é possível inferir que o tempo de uso do omeprazol aumenta proporcionalmente com a faixa etária. Além da média de idade, outra variável que teve diferença estatisticamente significativa foi a média de medicamentos, maior para aqueles pacientes que usam o omeprazol por mais de seis meses (4,8 medicamentos), comparado com os que utilizam omeprazol por menos tempo (3,8 medicamentos). Com relação às indicações de uso, houve diferença significativa para as indicações relacionadas com o tempo de uso. 
Tabela 3. Descrição da amostra conforme tempo de uso de omeprazol (até seis meses ou mais de seis meses).

\begin{tabular}{|c|c|c|c|c|}
\hline Variável & $\begin{array}{l}\text { Total n (\%) ou } \\
\text { média (DP) }\end{array}$ & $\begin{array}{l}\text { Até } 6 \text { meses n (\%) } \\
\text { ou média (DP) }\end{array}$ & $\begin{array}{l}\text { Mais de } 6 \text { meses n (\%) } \\
\text { ou média (DP) }\end{array}$ & $\mathbf{p}$ \\
\hline Média de idade & $64,6(15,1)$ & $59,3(3,8)$ & $66,3(13,3)$ & $0,003^{*}$ \\
\hline Média de medicamentos & $4,5(1,7)$ & $3,8(1,8)$ & $4,8(1,5)$ & $<0,0001^{*}$ \\
\hline Faixa etária & & & & $<0,0001^{*}$ \\
\hline 0 a 10 anos & $1(0,3 \%)$ & 0 & $1(0,4 \%)$ & \\
\hline 11 a 20 anos & $3(0,9 \%)$ & $3(6,3 \%)$ & 0 & \\
\hline 21 a 30 anos & $5(1,6 \%)$ & $2(4,2 \%)$ & $3(1,1 \%)$ & \\
\hline 31 a 40 anos & $17(5,4 \%)$ & $7(14,6 \%)$ & $10(3,7 \%)$ & \\
\hline 41 a 50 anos & $17(5,4 \%)$ & $3(6,3 \%)$ & $14(5,2 \%)$ & \\
\hline 51 a 60 anos & $60(18,9 \%)$ & $6(12,5 \%)$ & $54(20,1 \%)$ & \\
\hline 61 a 70 anos & $96(30,3 \%)$ & $14(29,2 \%)$ & $82(30,5 \%)$ & \\
\hline 71 anos ou mais & $118(37,2 \%)$ & $13(27,1 \%)$ & $105(39,0 \%)$ & \\
\hline Indicação de uso & & & & $<0,0001^{*}$ \\
\hline Doença do refluxo & $98(30,9 \%)$ & $6(12,5 \%)$ & $92(34,2 \%)$ & \\
\hline Úlcera gástrica & $48(15,1 \%)$ & $3(6,3 \%)$ & $45(16,7 \%)$ & \\
\hline Esofagite erosiva & $2(0,6 \%)$ & 0 & $2(0,7 \%)$ & \\
\hline Úlcera duodenal & $1(0,3 \%)$ & 0 & $1(0,4 \%)$ & \\
\hline Doença por Helicobacter pylori & $1(0,3 \%)$ & $1(2,1 \%)$ & 0 & \\
\hline Outras indicações** & $91(28,7 \%)$ & $26(54,2 \%)$ & $65(24,2 \%)$ & \\
\hline Sem justificativa & $76(24,0 \%)$ & $12(25,0 \%)$ & $64(23,8 \%)$ & \\
\hline
\end{tabular}

${ }^{*} p<0,05$ para o teste de qui-quadrado de Pearson para variáveis categóricas ou teste $t$ de Student para variáveis contínuas. ${ }^{* \star}$ Outras indicações, como registros de sintomas relacionados à necessidade de prescrição de omeprazol.

\section{Discussão}

Os dados apresentados no presente estudo indicam que houve maior prevalência de uso do omeprazol no sexo feminino, resultado que se repete em outros estudos relacionados ao tema. ${ }^{20} \mathrm{~A}$ média de medicamentos em uso foi de 4,5 por usuário, sendo maior em mulheres do que em homens. Quanto à idade, a média ficou em torno de 64 anos. Vários fatores colaboram para a alta prevalência de idosos entre os pacientes analisados, como o aumento da expectativa de vida e o aumento da utilização de serviços de saúde.

A média de idade e de medicamentos em uso por esses pacientes indicam que a polifarmácia aumenta com a progressão da idade, o que pode ser explicado por vários fatores, entre eles o aumento da morbidade. ${ }^{21} \mathrm{Em}$ muitos prontuários de idosos pôde ser verificado que não havia justificativa para a prescrição de omeprazol e, na maioria das vezes, por tempo prolongado. Desta forma, é possível inferir que alguns medicamentos podem ser indicados para os idosos sem haver clara correspondência entre a doença e a ação farmacológica, levando a um uso irracional de medicamentos nesta população. ${ }^{20}$

Com relação às doses utilizadas, foi possível observar que a maior parte dos pacientes que utilizam uma dose maior do que $20 \mathrm{mg}$ ao dia, dose diária recomendada para a maioria das indicações (úlcera duodenal ativa, doença do refluxo, tratamento de úlcera induzida por AINE) ${ }^{3}$, tem uma justificativa de uso no prontuário médico. Quanto a altas doses, os resultados do estudo apresentam maior prevalência no tratamento da doença do refluxo entre esses pacientes. 
A doença do refluxo foi a justificativa de uso mais descrita nos prontuários, com maior prevalência no sexo feminino, sendo esse resultado compatível com outro estudo realizado na Espanha. ${ }^{22}$ Em outros estudos publicados, além de indicarem que as mulheres são as mais atingidas por essa doença, os resultados dos mesmos apontam que isto pode estar relacionado ao sobrepeso, consumo excessivo de alimentos gordurosos, tabagismo e ao estresse. ${ }^{23}$

Com relação ao tempo de tratamento da DRGE, as análises realizadas mostram que a maioria dos usuários fazem tratamento com o omeprazol há mais de um ano. No entanto, o protocolo de tratamento para a doença do refluxo indica que o tempo ideal da terapêutica seria de 4 a 8 semanas. No caso de pacientes que não apresentem resposta totalmente satisfatória ao tratamento com IBP por 8 semanas, é possível ter a terapia prolongada por mais 4 semanas, antes de se determinar um insucesso terapêutico. Além disso, doses de manutenção com doses decrescentes até a mínima dose eficaz para manter o paciente assintomático e medidas comportamentais também são indicadas. ${ }^{2}$

A segunda indicação de uso de omeprazol mais prevalente foi a úlcera gástrica. A dose de omeprazol utilizada no tratamento da úlcera gástrica foi de $20 \mathrm{mg}$ ao dia para a maioria dos pacientes; no entanto, o tempo de tratamento, assim como na DRGE, foi prolongado, sendo que a maioria dos pacientes utilizavam omeprazol há mais de um ano.

O tempo de uso do omeprazol nos pacientes deste estudo foi prolongado, não apenas no tratamento da úlcera gástrica ou da DRGE, mas na população total estudada. Do total dos pacientes, 26,6\% utilizam o omeprazol há 2-5 anos e 25,8\% por mais de 5 anos.

Com relação às demais indicações de uso que foram citadas nos prontuários, como azia e dispepsia, e que representaram $26,9 \%$ das justificativas do uso do omeprazol, também apresentaram um tratamento prolongado. Isso pode indicar o uso deliberado desse medicamento sem investigação da causa primária que provoca os sintomas que o usuário refere ao médico.

A dispepsia, por exemplo, um dos sintomas de constante reclamação por parte dos pacientes, é a manifestação de diferentes doenças, entre elas, a DRGE, úlcera péptica gastroduodenal e dispepsia funcional, que devem ser diagnosticadas corretamente (ou tratadas com omeprazol usado como teste terapêutico), seguindo o correto protocolo de tratamento. ${ }^{24}$

Algumas características deste fármaco que podem colaborar para sua prescrição indiscriminada são sua alta eficiência e baixa toxicidade. No entanto, estudos alertam sobre os riscos relacionados ao uso prolongado de IBP, que envolvem riscos à saúde e à qualidade de vida dos usuários. Os profissionais prescritores devem considerar o risco-benefício quando necessário o uso prolongado destes medicamentos, assim como os demais profissionais da saúde devem estar atentos a sinais e sintomas relacionados aos efeitos adversos do uso prolongado dos IBP. Desta forma, ressalta-se a importância da atuação dos profissionais de saúde na promoção do uso racional de medicamentos e na oferta de informações à população sobre os riscos relacionados ao uso de IBP, principalmente de omeprazol, pela sua facilidade de acesso..$^{1,10}$

A interrupção do tratamento de longo prazo com omeprazol deve ser considerada com atenção. Revisão sistemática avaliando estratégias para o término de tratamentos com IBP verificou que a interrupção do uso do mesmo é viável desde que com acompanhamento médico, em que aqueles pacientes que utilizam omeprazol sem uma indicação clara podem reduzir ou interromper o tratamento com segurança. ${ }^{24}$ 
O estudo apresentado possui algumas limitações, entre elas, a restrição das receitas médicas avaliadas. Foram incluídas apenas as receitas provenientes de médicos da UBS em questão. Desta forma, pacientes da área adstrita desta UBS, mas que consultaram em outros serviços de saúde foram excluídos. Isso foi feito para assegurar que a prescrição do omeprazol estaria registrada no prontuário médico do paciente e, da mesma forma, a justificativa para essa prescrição.

Outra limitação deve-se ao fato de que as informações foram coletadas a partir dos prontuários, levando, muitas vezes, a informações incompletas. Em alguns casos, os pacientes haviam se cadastrado há pouco tempo na UBS, havendo poucas informações sobre as patologias, medicamentos em uso e sobre o histórico clínico desses pacientes. Por se tratar de um estudo utilizando dados de prontuário, não foi possível avaliar a percepção, bem como o papel do usuário e dos demais membros da equipe de saúde quanto ao uso racional de omeprazol.

\section{Conclusão}

Este estudo avaliou o perfil dos usuários de omeprazol, obtendo resultados em que foi possível observar a associação entre o maior tempo de uso deste fármaco com o maior número de medicamentos prescritos, indicação registrada em prontuário e idade elevada.

Embora mais estudos sejam necessários para elucidar as hipóteses mencionadas no presente trabalho, os resultados apontam para a necessidade do desenvolvimento de novos programas de ação para o uso racional de medicamentos, com foco na prescrição e uso adequado pelos usuários. Para isso, campanhas de caráter educativo, buscando a participação das entidades representativas dos profissionais de saúde, devem ser estimuladas pelos gestores do SUS. Deve ser dada atenção especial aos idosos nestes programas, tendo em vista que foram a população mais prevalente neste estudo, além de estar em crescimento no Brasil.

\section{Referências}

1. Chen J, Yuan YC, Leontiadis Gl, Howden CW. Recent safety concerns with proton pump inhibitors. J Clin Gastroenterol. 2012;46(2):93-114. DOI: http://dx.doi.org/10.1097/MCG.0b013e3182333820

2. Micromedex ${ }^{\circledR}$ Truven Health Analytics. V.2.0. DRUGDEX System. The Healthcare Business of Thomson Reuters, 2015. [acesso 8 Ago 2015]. Disponível em: http://www.micromedexsolutions.com/home/dispatch

3. Wolters Kluwer. Omeprazole: Drug Information. In: Up To Date. [acesso 10 Ago 2015]. Disponível em: http://www.uptodate. $\mathrm{com} /$ contents/omeprazoledruginformation?source=search_result\&search=omeprazole\&selectedTitle=1\%7E150

4. Brasil. Ministério da Saúde. Secretaria de Ciência, Tecnologia e Insumos Estratégicos. Departamento de Assistência Farmacêutica e Insumos Estratégicos. Uso Racional de Medicamentos: temas selecionados. Brasília: Ministério da Saúde; 2012.

5. Brasil. Ministério da Saúde. Relação Nacional de Medicamentos Essenciais: RENAME 2014. 9a ed. Brasília: Ministério da Saúde; 2015.

6. World Health Organization. WHO Model List of Essential Medicines. [acesso 4 Ago 2015]. Disponível em: http://www.who. int/selection_medicines/list/en/

7. Hoefler R, Leite BF. Segurança do uso contínuo de inibidores da bomba de prótons. Farmacoterapêutica. 2009;14(1-2):1-3.

8. Lima APV, Neto Filho MA. Efeitos em longo prazo de inibidores da bomba de prótons. Braz. J Surg Clin Res. 2013:5(3):45-9.

9. Caricol FC. Seguridad del omeprazol: ¿es adecuada la duración de los tratamientos? Farm Comunitarios. 2015;7(1):5-9. 
10. Wilhelm SM, Rjater RG, Kale-Pradhan PB. Perils and pitfalls of long-term effects of proton pump inhibitors. Expert Rev Clin Pharmacol. 2013;6(4):443-51. DOI: http://dx.doi.org/10.1586/17512433.2013.811206

11. García Rodríguez LA, Ruigómez A, Panés J. Use of acid-suppressing drugs and the risk of bacterial gastroenteritis. Clin Gastroenterol Hepatol. 2007;5(12):1418-23. DOI: http://dx.doi.org/10.1016/j.cgh.2007.09.010

12. Souza IKF, Silva AL, Araújo Júnior A, Santos FCB, Mendonça BPCK. Análise qualitativa das alterações anatomopatológicas na mucosa gástrica decorrentes da terapêutica prolongada com inibidores da bomba de prótons: estudos experimentais x estudos clínicos. Arq Bras Cir Dig. 2013;26(4):328-34. DOI: http://dx.doi.org/10.1590/S0102-67202013000400015

13. Yanagihara GR, de Paiva AG, Neto MP, Torres LH, Shimano AC, Louzada MJ, et al. Effects of long-term administration of omeprazole on bone mineral density and the mechanical properties of the bone. Rev Bras Ortop. 2015;50(2):232-8. DOI: http://dx.doi.org/10.1016/j.rbo.2014.05.012

14. Menegassi VS, Czeczko LEA, Czekzo LSG, loshi SO, Pisani JC, Ramos Junior O. Prevalência de alterações proliferativas gástricas em pacientes com uso crônico de inibidores de bomba de prótons. Arq Bras Cir Dig. 2010;23(3):145-9. DOI: http://dx.doi.org/10.1590/S0102-67202010000300003

15. Ameijeiras AH, González BC, Zúñiga VL. Prevalencia de prescripción-indicación de protectores gástricos en pacientes hospitalizados. Gac Sanit. 2007;21(5):412-5. DOI: http://dx.doi.org/10.1157/13110449

16. Palazón EM, Moreno CB. Inhibidores de la bomba de protones, ¿la protección mal entendida? FMC Form Med Contin Aten Prim. 2011;18(5):243-5.

17. Centro de Informação sobre Medicamentos. Uso Racional de omeprazol. Alerta terapêutico - Junho 2009. [acesso 10 Ago 2015]. Disponível em: http://www.prefeitura.sp.gov.br/cidade/secretarias/upload/saude/arquivos/assistenciafarmaceutica/ omeprazol.pdf

18. Patterson SM, Cadogan CA, Kerse N, Cardwell CR, Bradley MC, Ryan C. Interventions to improve the appropriate use of polypharmacy for older people. Cochrane Database Syst Rev. 2014;10:CD008165.

19. Schroeter G, Chaves LL, Engroff P, Faggiani FT, DE Carli GA, Morrone FB. Estudo de utilização de anti-ulcerosos na população Idosa de Porto Alegre, RS, Brasil. Rev HCPA. 2008;28(2):89-95.

20. López-Dóriga Bonnardeaux P, Neira Álvarez M, Mansilla Laguía S. Inhibidores de la bomba de protones: estudio de prescripción en una Unidad de Recuperación Funcional. Rev Esp Geriatr Gerontol. 2013;48(6):269-71. DOI: http://dx.doi. org/10.1016/j.regg.2013.07.004

21. Rozenfeld S. Prevalência, fatores associados e mau uso de medicamentos entre os idosos: uma revisão. Cad Saúde Pública. 2003;19(3):717-24. DOI: http://dx.doi.org/10.1590/S0102-311X2003000300004

22. Moraes-Filho JP, Chinzon D, Eisig JN, Hashimoto CL, Zaterka S. Prevalence of heartburn and gastroesophageal reflux disease in the urban Brazilian population. Arq Gastroenterol. 2005;42(2):122-7. PMID: 16127569 DOI: http://dx.doi. org/10.1590/S0004-28032005000200011

23. Chang P, Friedenberg F. Obesity and GERD. Gastroenterol Clin North Am. 2014;43(1):161-73. PMID:24503366 DOI: http:// dx.doi.org/10.1016/j.gtc.2013.11.009

24. Haastrup P, Paulsen MS, Begtrup LM, Hansen JM, Jarbøl DE. Strategies for discontinuation of proton pump inhibitors: a systematic review. Fam Pract. 2014;31(6):625-30. DOI: http://dx.doi.org/10.1093/fampra/cmu050 